\title{
Mapping routine malaria incidence at village level for targeted control in Papua New Guinea
}

\author{
Daniela Rodríguez-Rodríguez, ${ }^{1-3}$ Seri Maraga, ${ }^{3}$ Sharon Jamea-Maiasa, ${ }^{3}$ Anthony Tandrapah, ${ }^{3}$ \\ Leo Makita, ${ }^{4}$ Peter M. Siba, ${ }^{3}$ Ivo Mueller, ${ }^{5,6}$ Justin Pulford, ${ }^{7}$ Manuel W. Hetzel ${ }^{1,2}$ \\ ${ }^{1}$ Department of Epidemiology and Public Health, Swiss Tropical and Public Health Institute, Basel, \\ Switzerland; ${ }^{2}$ University of Basel, Basel, Switzerland; ${ }^{3}$ Papua New Guinea Institute of Medical Research, \\ Goroka and Madang, Papua New Guinea; ${ }^{4}$ National Department of Health, Waigani, Papua New Guinea; \\ ${ }^{5}$ Walter and Eliza Hall Institute of Medical Research, Melbourne, Australia; ${ }^{6}$ Pasteur Institute, Paris, \\ France; ${ }^{7}$ Liverpool School of Tropical Medicine, Liverpool, United Kingdom
}

\author{
Correspondence: Daniela Rodríguez-Rodríguez, Department of \\ Epidemiology and Public Health, Swiss Tropical and Public Health \\ Institute, Socinstrasse 57, 4002 Basel, Switzerland. \\ Tel. +41.61.284.86.00. \\ E-mail: daniela.rodriguez@swisstph.ch
}

Key words: Surveillance; Malaria; Papua New Guinea; Malaria control; Surveillance-response; Visualisation.

Acknowledgements: the authors would like to thank all patients and communities who agreed to participate in this study. We are grateful to the study nurses who collected data and samples at the sentinel health facilities (Mrs. Naeobo Oren in East Cape, Mr. Paul Basanu in Karimui, Ms. Rhona Sao in Sausi, and Mrs. Lia Pitan in Lemakot), and to the authorities and organisations hosting them. We thank the Papua New Guinea Institute of Medical Research census field teams, the data managers and data entry staff and acknowledge the support of the National Department of Health through Mr. Leo Makita and his team. We thank Mr. Andreas Weber and Point de Vue for their support and enthusiasm. Their collaboration made the audio-visualisation of this paper possible.

Contributions: MWH, JP, IM, LM and PMS conceived the study. SM, SJM, AT, MWH and JP established and supervised data collection systems and LM facilitated access to the study sites. DRR analysed the data and drafted the manuscript and video concept with inputs from MWH. All authors reviewed and approved the final manuscript and video.

Conflict of interest: the authors declare no potential conflict of interest.

Funding: funding for data collection was provided through a Global Fund to Fight AIDS, Tuberculosis and Malaria grant to Papua New Guinea. DRR was supported by the Forlen Stiftung, Basel, Switzerland, and the R. Geigy Foundation, Basel, Switzerland.

Received for publication: 11 July 2019.

Revision received: 23 August 2019.

Accepted for publication: 10 September 2019.

${ }^{\circ}$ Copyright: the Author(s), 2019

Licensee PAGEPress, Italy

Geospatial Health 2019; 14:798

doi:10.4081/gh.2019.798

This article is distributed under the terms of the Creative Commons Attribution Noncommercial License (CC BY-NC 4.0) which permits any noncommercial use, distribution, and reproduction in any medium, provided the original author(s) and source are credited.

\begin{abstract}
Malaria surveillance and response-systems are essential for identifying the areas most affected by malaria and for targeting interventions and optimising resources. This study aimed to assess whether the visualisation of routinely collected health facility data linked to village of residence provides evidence for targeting control interventions in four sentinel health facilities in Papua New Guinea. A video format was used to visualise the dynamics in case incidence over time and space alongside photographs illustrating the context of the data collection in the study sites. Incidence changes overtime were illustrated in animated maps. Despite limitations, this approach appeared useful in sites with very few remaining cases or with increasingly marked heterogeneity. Villages that could benefit from targeted interventions or investigations were identified.
\end{abstract}

Video link: https://www.youtube.com/watch?v=KISbow_E07I

\section{Background}

Variation in the risk of malaria prevalence and incidence between villages in regions with on-going transmission has long been recognised (Greenwood, 1989; Bousema et al., 2012). Such variations become more evident in regions with moderate and low transmission, e.g. after scale-up of malaria control (Bousema et al., 2012). Malaria control efforts in Papua New Guinea (PNG) were re- intensified in 2004. Differences in malaria burden, transmission, and impact of interventions have subsequently been identified even between neighbouring villages (Hetzel et al., 2014, 2016), confirming earlier findings of small-area heterogeneity (Cattani et al., 1986). An analysis of routine health-facility data from seven sentinel sites found that reductions in malaria incidence were associated with Long Lasting Insecticidal Nets distributions but the effect varied between sites (Rodríguez-Rodríguez et al., 2019, in press). As malaria transmission decreases, and resources remain limited, targeting of interventions becomes increasingly important (Mendis et al., 2009; Bousema et al., 2012). Malaria surveillance and response-systems are essential for identifying the areas or population groups that are most affected by malaria and for targeting resources for maximum impact (WHO, 2018). The Global Technical Strategy for Malaria 20162030 proposes the use of a comprehensive approach that includes vector control measures and early diagnosis and treatment, espe- 
cially at the village level (WHO 2015, 2018). Identifying villages with on-going transmission and monitoring changes over time is therefore of utmost importance to effectively target interventions. Particularly in settings with weak health systems this information should be generated by simple approaches. Routine data collected at health facilities may provide a viable option if the relevant information is captured and readily analysed. However, research on how to best apply geospatial analyses of simple routine data to identify heterogeneity at village level and support targeting malaria interventions is scarce (Kelly et al., 2012).

This study aimed to assess whether the visualisations of health facility data linked to village of residence of patients provides evidence for targeting malaria control interventions. It used malaria incidence data linked to the self-reported village of residence of the patients, collected routinely in four sentinel health facilities (SHF) in PNG. If found to be a valid approach, the operational feasibility of targeting malaria control at district or sub-district level would have to be investigated within the frame of existing capacities and resources of the local health system.

Sentinel surveillance was established in the health centres of East Cape (Southern Region), Sausi (Momase), Karimui (Highland) and Lemakot (Islands) - one SHF per geographical region of PNG. Surveillance was established as part of the continuous independent evaluation of the National Malaria Control Program (NMCP) (Hetzel et al., 2012, 2014, 2015). Details of the sites are provided elsewhere (Hetzel et al., 2014; RodríguezRodríguez et al., 2019, in press). All outpatient cases attending the SHF were routinely screened for a history of fever during the previous three days. A study nurse at the facility collected a capillary blood sample from all consenting fever patients of all ages for diagnosis of malaria by Rapid Diagnostic Test (RDT; ICT Malaria Combo HRP2/aldolase, ICT Diagnostics, South Africa) Demographic details including village of residence and self-reported mosquito-net use the previous night were recorded on paper case report forms alongside RDT results. Paper forms were then double entered at the Papua New Guinea Institute of Medical Research (PNGIMR). The study team ensured availability of RDTs throughout the surveillance period.

Data was collected from 2010 to 2014 to characterise annual incidence variations between villages in the catchment areas of the
SHFs and identify patterns that could guide malaria control efforts. Recently, a paper-based Malaria Register has been implemented by the PNG National Department of Health and an electronic National Health Information System (eNHIS) is being piloted (Rosewell et al., 2017). The register and eNHIS routinely collect malaria indicators linked to the village of residence of the patient, which is comparable to this analysis, allowing the scale-up of the approach if it proves useful.

The delineation of the SHF catchment areas was defined by local health authorities. A population census conducted by the PNGIMR in the SHF catchment areas at baseline, during which all houses were identified and each household member was listed, was used as source of village coordinates and population denominator data (Hetzel et al., 2014). The geo-referenced year 2000 National Census database was used to complement the PNGIMR census, particularly to identify villages outside the catchment area. During the surveillance period 25,097 fever cases were tested for malaria across all SHF, 38\% (95\% CI: 37.6-38.8) were RDT-positive. Table 1 details the number of malaria cases diagnosed at the SHF residing within and outside the SHF catchment area. The analysis includes only the cases within the catchment area. It is important to note that a large number of patients from outside the catchment area were diagnosed and treated in East Cape and Lemakot SHFs. Both Health Centres are located in areas of constant transit of people. The video format allowed us to visualise the dynamics in case incidence over time and space alongside photographs illustrating the context of the data collection in the study sites. The main focus of the visualisation was on the animated maps illustrating incidence changes overtime. The audio-visual format is easily accessible to a range of stakeholders with the potential to better communicate geospatial relationships in an understandable format (Krieger et al., 2012).

Aggregated annual malaria incidence was calculated for every village with at least one malaria case reported by the SHFs. Annual incidence in children under five years of age was calculated and mapped as a proxy of local transmission since young children are the least immune age group and compared to adults are less likely to travel (Bousema et al., 2012). Self-reported mosquito-net use in all fever patients during the last year of surveillance (2014) was mapped by village and reported net use in

Table 1. Total number of malaria cases (all ages) and malaria cases in children under five years of age residing within and outside the catchment area.

\begin{tabular}{llccc} 
Site & Age & $\begin{array}{c}\text { Malaria cases residing } \\
\text { in the catchment area }\end{array}$ & $\begin{array}{c}\text { Malaria cases residing } \\
\text { outside the catchment area }\end{array}$ & N (\%) \\
East Cape & All ages & $3,265(93)$ & $250(7)$ & $3,515(100)$ \\
& Children $<5$ years & $1,076(93)$ & $82(7)$ & $1,158(100)$ \\
Sausi & All ages & $1,532(99)$ & $13(1)$ & $1,545(100)$ \\
& Children $<5$ years & $305(98)$ & $6(2)$ & $311(100)$ \\
\hline \multirow{2}{*}{ Karimui } & All ages & $545(99)$ & $5(1)$ & $553(100)$ \\
& Children $<5$ years & $260(98)$ & $1,142(29)$ & $265(100)$ \\
\multirow{2}{*}{ Lemakot } & All ages & $2,831(71)$ & $299(27)$ & $3,973(100)$ \\
& Children $<5$ years & $818(73)$ & $1,413(15)$ & $1117(100)$ \\
\hline \multirow{2}{*}{ Total } & Total cases in all ages & $8,173(85)$ & $392(14)$ & $9,586(100)$ \\
& Total cases in children $<5$ years & $2,459(86)$ & $2,851(100)$ \\
\hline
\end{tabular}


all fever cases was graphed by year as an indicator of trends over time. Following a general declining trend, clear differences in incidence between villages were found in some sites. Mapping of village-level incidence appeared most useful in settings with very few malaria cases (Karimui and Sausi) or with pronounced spatial clustering of cases (Lemakot). In such settings, villages that could benefit from targeted interventions could be identified. However, further investigations in some of the identified local foci are required to understand local heterogeneity. Unequal access to health facilities, availability of other health care providers and treatment seeking behaviour may confound village-level incidence particularly if data is only originating from one facility. In addition, in some communities, village, hamlet and ward names may be used inconsistently by both patients and health workers. It is possible that cases in small communities are attributed to larger nearby villages. Furthermore, case-reporting becomes inaccurate in areas with constant transit of people. Uniform surveillance across all health facilities and a harmonised use of village names could optimise the current approach. Villages have been used as operational units for household-level net distributions in PNG. Villages may also be the smallest feasible unit to target interventions making village level data highly relevant. Since effective management of malaria programmes requires geo-spatial components to inform response-systems, a next step could devise a simple standardised approach to generate spatial data on malaria risk that can be easily translated into response action can complement universal coverage campaigns in a meaningful way. Since 2015, eNHIS has been piloted in 184 health facilities in PNG. The platform includes a geo-referencing feature for mapping malaria cases at a village level and automated data analysis, reporting and identification of outbreaks (Rosewell et al., 2017). If proven successful eNHIS could considerably strengthen malaria surveillance in PNG. A similar Spatial Decision Support System building upon and extending existing data collection systems and exploiting current geo-spatial tools has been validated in nearby Vanuatu and Solomon Islands in areas of very low transmission (Kelly et al., 2012). Areas in PNG that have reached a low level of transmission with clear foci may benefit from a similar approach. However, local (sub-national) capacity to further investigate local foci and implement targeted response action would be required as much as sufficient and sustained funding for these activities.

\section{Outlook}

- Age-specific malaria data collected routinely at health facilities and linked to the village of residence of patients may direct programmes to local foci of transmission.

- This approach appears most useful in settings with few cases or marked heterogeneity, where it may direct further investigations or complementary interventions.

- A simple tool for calculating and mapping malaria case incidence at district or sub-district level, as is currently included in the piloted eNHIS, is required to operationalise the approach, along with the capacity, policies, and mechanisms required to implement targeted response action at the respective operational level.

- The chosen audio-visual format is easily accessible to a range of stakeholders with the potential to better communicate geospatial relationships in an understandable format.

\section{Overall aim}

To assess weather, the visualisation of health information (malaria incidence, net use and residence of patients) extracted from a routinely implemented surveillance system can inform local malaria control programmes to better target interventions. Malaria surveillance systems are crucial for identifying the areas that are most affected by malaria. The proposed approach adds a geospatial component to health facility data in order to understand differences in malaria burden between villages and identify communities that would benefit from targeted complementary interventions.

\section{Software}

Maps were generated using the open-source software QGIS (version 3.0 Girona). The video was edited using Adobe Premier Pro CC (version 13.0.2, Adobe Systems Incorporated, San Jose, CA, USA).

\section{References}

Bousema T, Griffin JT, Sauerwein RW, Smith DL, Churcher TS, Takken W, Ghani A, Drakeley C, Gosling R, 2012. Hitting hotspots: spatial targeting of malaria for control and elimination. PLoS Med 9:1-7.

Cattani JA, Moir JS, Gibson FD, Ginny M, Paino J, Davidson W, Alpers MP, 1986. Small-area variations in the epidemiology of malaria in Madang Province. PNG Med J 29:11-7.

Greenwood BM, 1989. The microepidemiology of malaria and its importance to malaria control. Trans R Soc Trop Med Hyg 83:259.

Hetzel MW, Gideon G, Lote N, Makita L, Siba PM, Mueller I, 2012. Ownership and usage of mosquito nets after four years of largescale free distribution in Papua New Guinea. Malar J 11:192.

Hetzel MW, Morris H, Tarongka N, Barnadas C, Pulford J, Makita L, Siba PM, Mueller I, 2015. Prevalence of malaria across Papua New Guinea after initial roll-out of insecticide-treated mosquito nets. Trop Med Int Health 20:1745-55.

Hetzel MW, Pulford J, Maraga S, Barnadas C, Reimer LJ, Tavul L, Jamea-Maiasa S, Tandrapah T, Maalsen A, Makita L, Siba PM, Mueller I, 2014. Evaluation of the Global Fund-supported National Malaria Control Program in Papua New Guinea, 2009-2014. PNG Med J 57:7-29.

Hetzel MW, Reimer LJ, Gideon G, Koimbu G, Barnadas C, Makita L, Siba PM, Mueller I, 2016. Changes in malaria burden and transmission in sentinel sites after the roll-out of long-lasting insecticidal nets in Papua New Guinea. Parasite Vectors 9:340.

Kelly GC, Tanner M, Vallely A, Clements ACA, 2012. Malaria elimination: moving forward with spatial decision support systems. Trends Parasitol 28:297-304.

Krieger GR, Bouchard MA, de Sa IM, Paris I, Balge Z, Williams D, Singer BH, Winkler MS, Utzinger J, 2012. Enhancing impact: visualization of an integrated impact assessment strategy. Geospat Health 6:303-6.

Mendis K, Rietveld A, Warsame M, Bosman A, Greenwood BM, Wernsdorfer WH, 2009. From malaria control to eradication: the WHO perspective. Trop Med Int Health 14:802-9.

Rodríguez-Rodríguez D, Marga S, Lorry L, Robinson LJ, Siba PM, 
Mueller I, Pulford J, Ross A, Hetzel MW, 2019. Repeated mosquito net distributions, improved treatment, and trends in malaria cases in sentinel health facilities in Papua New Guinea. Malar J [in press].

Rosewell A, Makita L, Muscatello D, John LN, Bieb S, Hutton R, Ramamurthy S, Shearman P, 2017. Health information system strengthening and malaria elimination in Papua New Guinea. Malar J 16:1-10.

WHO, 2015. Global Technical Strategy for Malaria 2016-2030. World Health Organization, Geneva, Switzerland.

WHO, 2018. World Malaria Report 2018. World Health Organization, Geneva, Switzerland. 